\title{
Influence of Leukocytospermia on Semen Parameters and the Outcome of Intracytoplasmic Sperm Injection
}

\author{
Hela Hannachi ${ }^{1}$, Hanen Elloumi ${ }^{2}$, Salma Ben Khlifa ${ }^{3}$, Manel Hamdoun ${ }^{4}$, Khadija Kacem ${ }^{5}$, Olfa Bahri ${ }^{6}$
}

\begin{abstract}
Background: Leukocytospermia (LCS) is implicated in male infertility since it was found in $30 \%$ of the infertile male. However, its influence on semen parameters remains controversial.

Aims and objectives: This study aimed to determine the impact of LCS on semen parameters and intracytoplasmic sperm injection (ICSI) results in infertile couples.

Materials and methods: A retrospective clinical study was conducted at Aziza Othmana Hospital of Tunis. Sperm parameters from infertile men were studied for volume, motility, vitality, and morphology according to seminal leukocytes. Intracytoplasmic sperm injection results were also compared to those observed in a control group matched by age, ICSI indication, hormonal profile, and endometrium thickness.

Results: No statistical difference was observed for all semen characteristics except the volume which was higher for the non-leukospermic group $(p=0.035)$.

Conclusion: Intracytoplasmic sperm injection outcomes were lower in the leukospermic group than the controls but the difference was not statistically significant.

Keywords: Intracytoplasmic sperm injection, Leukocytospermia, Male infertility, Semen parameters.

International Journal of Infertility and Fetal Medicine (2020): 10.5005/jp-journals-10016-1202
\end{abstract}

\section{INTRODUCTION}

Leukocytospermia (LCS) or pyospermia is observed in $10-32 \%$ of infertile men; it is defined, by the World Health Organization (WHO), as the presence of at least one million leukocytes per milliliter of semen (WHO, Manual 2010). Leukocytospermia is considered as an indicator of genital tract infection or inflammation; that is why it is one of the evaluated parameters in the seminal analysis when male infertility is diagnosed. ${ }^{1}$ This association remains controversial since high concentrations of semen leukocytes were also reported in the absence of inflammatory symptoms or microbial infection. ${ }^{2}$

Although semen leukocytes are essential to remove pathogens and abnormal cells from seminal fluid, their presence at high concentrations can hurt semen quality. Some studies suggested that LCS decreased sperm motility and count while others reported a high frequency of altered spermatozoon associated with LCS. ${ }^{3}$ Leukocytospermia results in high concentrations of reactive oxygen species (ROS) produced by leukocytes which can lead to sperm damage via membrane alterations, DNA fragmentation, and protein inactivation. ${ }^{4,5}$

Few and contradictory reports were published about the influence of LCS on the outcomes of in vitro fertilization (IVF)/ intracytoplasmic sperm injection (ICSI). Some authors reported a reduced fecundation rate associated with LCS. ${ }^{6}$ Conversely, no negative influence of LCS on fertilization and pregnancy rates following IVF or ICSI was observed in other studies. ${ }^{7}$ This is the first Tunisian study conducted to evaluate the impact of LCS in semen parameters and its influence on the outcomes of ICSI.

\section{Materials and Methods}

\section{Studied Population}

This retrospective study was conducted for 9 months; it included 60 patients undergoing ICSI at the assisted reproduction unit
${ }^{1,6}$ Department of Microbiology and Biochemistry Laboratory, Faculty of Medicine of Tunis, Aziza Othmana Hospital, Tunis, Tunisia

${ }^{2}$ Department of Reproductive Medicine Laboratory, Fertilia Center LA Rose, Tunis, Tunisia

${ }^{3}$ Biology Department, International Hospital of Carthagene, Tunis, Tunisia

${ }^{4}$ Microbiology and Biochemistry Department, Aziza Othmana Hospital, Tunis, Tunisia

${ }^{5}$ Department of Reproductive Medicine Laboratory, Faculty of Medicine of Tunis, Aziza Othmana Hospital, Tunis, Tunisia

Corresponding Author: Hela Hannachi, Department of Microbiology and Biochemistry Laboratory, Faculty of Medicine of Tunis, Aziza Othmana Hospital, Tunis, Tunisia, Phone: +216255551028, e-mail: helahanrejeb@gmail.com

How to cite this article: Hannachi $\mathrm{H}$, Elloumi $\mathrm{H}$, Ben Khlifa $\mathrm{S}$, et al. Influence of Leukocytospermia on Semen Parameters and the Outcome of Intracytoplasmic Sperm Injection. Int J Infertil Fetal Med 2020;11(2):33-36.

Source of support: This study was supported by the "Tunisian secretariat for Scientific Research and Technology" (SERST, contract: LR16SP01).

Conflict of interest: None

of Aziza Othmana Hospital (Tunis, Tunisia). All patients were asymptomatic for genitourinary infections and had been screened for Chlamydia, genital Mycoplasma, and other bacterial infections during follow-up. All patients with a history of smoking, drug consummation, toxin exposure, azoospermia, genital disorders, such as, varicocele, testicular abnormalities, and systemic diseases affecting fertility were excluded. The studied population was subdivided into two groups after sperm analysis according to the presence or the absence of LCS. These two groups were matched 
by partners' age, ICSI indication, wives' hormonal status, and protocol stimulation ovarian response. The study was approved by an institutional ethics committee. Written informed consent was obtained from all patients.

\section{Semen Analysis}

Semen sample was collected by masturbation from all patients after 2-5 days of abstinence. It was liquefied by an incubation, for 30 minutes at $35^{\circ} \mathrm{C}$ before analysis. Semen analysis was performed for the standard quality parameters according to the WHO guidelines (WHO Manual 2010). Round cells and leukocytes were estimated by a counting cell.

\section{ICSI Procedure}

At first, hormonal stimulation protocol was performed for partners according to underlying pathology. Monitoring was performed by ultrasound and serum estradiol levels. Oocyte retrieval, collection, and preparation of the sperm collection, culture, evaluation, and transfer of embryo were performed as described previously by Daya et al. ${ }^{8}$ An eventual pregnancy was confirmed when serum beta-hCG titer was $\geq 100 \mathrm{mUI} / \mathrm{mL}, 10$ days after embryo transfer.

Intracytoplasmic sperm injection results were evaluated by the index of sperm selection, fecundity and cleavage rates, topquality embryos, and pregnancy rates per oocyte retrieval and embryo transfer.

The index of sperm selection is the proportion of final selected spermatozoa from the initial concentration. The fecundity rate was defined by the ratio of the attained zygote to microinjected oocytes. Top-quality embryo corresponded to an embryo containing 4 equal blastomeres and $<10 \%$ of fragments.

\section{Statistical Analysis}

it was performed by IBM SPSS 17.0 software (SPSS, Inc., IBM, Armonk, NY, USA). The student's $t$-test was used to compare means between different groups. Fisher's exact test was used to compare percentages. Values were considered significant when $p$ values were $\leq 0.05$.

\section{Results}

\section{Baseline Characteristics of the Two Groups}

The mean age was 39 years for all patients and 33 years for their partners. Male infertility was reported in $48 \%$, female infertility in $32 \%$ and no etiology was found in $20 \%$ of cases. A comparison between leukospermic and non-leukospermic groups is reported in Table 1. No statistically significant difference was observed in the baseline characteristics between the two groups. There is also no statistical difference for the medium number of retrieved and mature oocytes.

\section{Semen Parameters}

Table 2 reports a comparison between the two groups according to sperm parameters. No statistical difference was observed for concentration, motility, vitality, and rate of typical form for spermatozoa. The medium volume of collected sperm was higher for the non-leukospermic group ( $p=0.035)$.

\section{ICSI Outcomes}

Before ICSI, sperm preparation efficiency was 15.3 and $13.2 \%$ in the two groups, respectively. A comparison between them for ICSI outcomes is reported in Table 3. All studied parameters were lower for the leukospermic group than for the controls but a difference was not statistically significant.
Table 1: Comparison between leukospermic and non-leukospermic groups for baseline parameters

\begin{tabular}{llll}
\hline & $\begin{array}{l}\text { Leukospermic group } \\
(n=30)\end{array}$ & $\begin{array}{l}\text { Non-leukospermic } \\
\text { group }(n=30)\end{array}$ & pvalue \\
\hline $\begin{array}{l}\text { Female age } \\
\text { (years) }\end{array}$ & $33.22 \pm 5.39$ & $33.58 \pm 4.37$ & NS \\
$\begin{array}{l}\text { Male age (years) } \\
\text { Type of infertility }\end{array}$ & $39.32 \pm 6.62$ & $39.16 \pm 3.89$ & NS \\
$\begin{array}{l}\text { Male infertility } \\
\text { Female infertility }\end{array}$ & $43 \%$ & $52 \%$ & NS \\
$\begin{array}{l}\text { Unexplained } \\
\text { etiology }\end{array}$ & $22 \%$ & $28 \%$ & NS \\
$\begin{array}{l}\text { Serum FSH level } \\
\text { at day 3 (mUl/mL) }\end{array}$ & $6.2 \pm 2.53$ & $20 \%$ & NS \\
$\begin{array}{l}\text { Serum LH level at } \\
\text { day 3 (mUl/mL) }\end{array}$ & $3.77 \pm 1.83$ & $6.28 \pm 1.66$ & NS \\
$\begin{array}{l}\text { Serum } \mathrm{E}_{2} \text { level at } \\
\text { 1st day of ovula- }\end{array}$ & $2,151 \pm 1,064.96$ & $1,716 \pm 1,025.88$ & NS \\
$\begin{array}{l}\text { tion triggering } \\
\text { (pg/mL) }\end{array}$ & & & NS \\
$\begin{array}{l}\text { Number of oo- } \\
\text { cytes retrieved } \\
\text { Number of ma- } \\
\text { ture oocytes }\end{array}$ & $8.6 \pm 3.65$ & $8.57 \pm 5.75$ & NS \\
$\begin{array}{l}\text { Endometrium } \\
\text { thickness (mm) }\end{array}$ & $10.31 \pm 2.36$ & $10.61 \pm 2.16$ & NS \\
\hline & & & NS \\
\hline
\end{tabular}

Table 2: Comparison between the leukospermic group and nonleukospermic groups for semen parameters

\begin{tabular}{lccl}
\hline & $\begin{array}{l}\text { Leukospermic } \\
\text { group }\end{array}$ & $\begin{array}{l}\text { Non-leukosper- } \\
\text { mic group }\end{array}$ & p value \\
\hline $\begin{array}{l}\text { Volume }(\mathrm{mL}) \\
\begin{array}{l}\text { Concentration } \\
\text { (million/mL) }\end{array}\end{array}$ & $2.04 \pm 1.29 \pm 30.91$ & $29.9 \pm 1.63$ & 0.035 \\
$\begin{array}{l}\text { Progressive } \\
\text { mobility }\end{array}$ & $25.68 \pm 17.52$ & $31 \pm 17.14$ & 0.232 \\
$\begin{array}{l}\text { Typical forms } \\
\text { percentage (\%) }\end{array}$ & $29 \pm 16.06$ & $35 \pm 24.61$ & 0.386 \\
Vitality (\%) & $53.63 \pm 27.61$ & $62.5 \pm 23.38$ & 0.414 \\
\hline
\end{tabular}

Table 3: Comparison between the leukospermic group and nonleukospermic groups for ICSI outcomes

\begin{tabular}{llll}
\hline & $\begin{array}{l}\text { Leukospermic } \\
\text { group }\end{array}$ & $\begin{array}{l}\text { Non-leukosper- } \\
\text { mic group }\end{array}$ & p value \\
\hline Fecundity rate (\%) & $56.47 \pm 0.32$ & $65.27 \pm 0.36$ & 0.311 \\
Cleavage rate (\%) & $67.7 \pm 0.33$ & $78 \pm 0.31$ & 0.206 \\
$\begin{array}{l}\text { Top embryos (\%) } \\
\text { Pregnancy rate/ }\end{array}$ & $37.39 \pm 0.38$ & $42.06 \pm 0.38$ & 0.629 \\
$\begin{array}{l}\text { oocyte retrieval (\%) } \\
\begin{array}{l}\text { Pregnancy rate/em- } \\
\text { bryo transfer (\%) }\end{array}\end{array}$ & 33.33 & 37.5 & 0.627 \\
\hline
\end{tabular}

\section{Discussion}

The results of this study did not show any association between LCS and alterations in sperm parameters; the only volume of semen samples was lower when high concentrations of leukocytes were observed probably due to an inflammation in reproductive male 
ducts. Up to now, the impact of LCS on sperm function is still debated. Some authors reported a significant increase in semen hyperviscosity in the LCS group, attributed to the inflammation associated with male accessory gland infection, dysfunction of the sex glands, or the immune response. ${ }^{9}$ Others observed a significant decrease in sperm motility, concentration, and morphology. ${ }^{3}$ Conversely, no significant difference was reported by several studies between samples with or without LCS. ${ }^{7}$ These divergent results could be attributed to methods used for leukocytes quantification which are poorly correlated especially at low concentrations of cells. In this study, we used morphologic evaluation based on microscopic cytology; this method is simple and reproducible but it is considered a subjective one. The gold standard recommended by $\mathrm{WHO}$ is the peroxidase test. However, compared to immunocytochemical techniques or flow cytometry, this method has a low sensitivity ranged from 47 to $68 \% .{ }^{10}$ Cavagna et al. had previously shown that a significant percentage of patients diagnosed with LCS by flow cytometry are wrongly classified as non-LCS by the peroxidase test. ${ }^{11}$ Indirect immunofluorescence was also used for cell quantification; it is reported to be a reliable technique with a limited risk of false-positive. ${ }^{12}$ Nowadays, flow cytometry is considered the most precise method; however, its high cost and the necessity of specific operational expertise limit its use in routine. ${ }^{13}$ In addition, the threshold used to define LCS seems to contribute to discordant results observed for the impact of LCS in sperm parameters; that is why the value of one million proposed by WHO is criticized by different authors. ${ }^{14}$ Some of them considered it too high; others proposed to decrease it to 20,000 leukocytes/ $\mathrm{mL} .{ }^{15}$ Recently, it was suggested that the presence of leukocytes below the WHO threshold is associated with the deterioration of sperm quality. ${ }^{14,16}$ In reality, LCS probably compromises indirectly sperm motility and viability by increasing levels of nitric oxide (NO). The concentration of this free radical increased when the activity of NO synthase was affected in presence of LCS. ${ }^{11}$

We did not observe any statistically significant differences between the LCS and non-LCS groups for fecundity rate, pregnancy rates, and top-quality embryos; these results suggest the absence of a negative impact for LCS on ICSI outcomes. Our results are in agreement with those published by Lackner et al. and Cavagna et al. who found comparable implantation rate (18 vs $10 \%, p=0.10$ ) and clinical pregnancy rate ( 25 vs $14 \%, p=0.13$ ) with or without LCS. $^{7,11}$ Moreover, a recent meta-analysis, conducted to analyze the relationship of LCS with fertilization rate and with the occurrence of pregnancies after assisted reproduction techniques (ART), confirmed the absence of adverse effects of LCS on the reproductive potential after ART. ${ }^{17}$ However, it is important to notify that leukocytes are the main source of inflammatory mediators and ROS are important to spermatozoon capacitation and hyperactivation. ${ }^{18}$ Their presence in high concentration can lead, via ROS cocultured inadvertently with spermatozoon during ART, to sperm damage with a negative effect on oocyte fertilization and embryonic development. ${ }^{19,20}$ Alteration of DNA integrity was described particularly for men with severe oligoasthenospermia. ${ }^{21}$ That is why, some authors proposed to perform sperm preparation before IVF by reducing the time of sperm centrifugation, using a fresh sample, and centrifugation on density gradient; these methods can lead to reduce ROS by removal of leukocytes and scrap cells. ${ }^{6,22}$ It was also reported that in addition to the incubation medium, antioxidants like vitamins $\mathrm{C}$ and $\mathrm{E}$, glutathione, hypotaurine, albumin, or $\mathrm{N}$-acetyl cysteine protect spermatozoon from DNA alteration and lipid peroxidation. ${ }^{18,23-27}$ In some assisted medical procreation centers, a selected sperm injection technique (IMSI) is used; this procedure, based on sperm normality, is capable to bypass the negative effects of LCS and consequences of ROS on sperm quality. ${ }^{28}$ However, the possibility that the presence of leukocytes is a benefit to ART outcomes should be considered. According to Barraud-Lange et al., LCS was associated with increasing fertilization rate, cleavage rate, clinical pregnancy rate, gestational age, and mean infant weight for 3,508 IVF/ICSI cycles. ${ }^{29}$

In conclusion, the results of this first Tunisian study indicate that LCS has no impact on sperm quality and ICSI outcomes. However, a further trial with a larger sample size and targeting inflammatory markers such as cytokines should be performed to confirm these results. DNA fragmentation should be also studied to know if it is interesting to use it as a prognosis marker for LCS patients who are undergoing IVF.

\section{References}

1. Krause W, Herbstreit F, Slenzka W. Are viral infections the cause of leukocytospermia? Andrologia 2002;34(2):87-90. DOI: 10.1046/j.03034569.2001.00484.x.

2. Chen L, Yu SL, Rajesh H. Is semen polymorphonuclear leucocytes count a good predictor of male genital tract infection? Singapore Med J 2013;54(6):328-331. DOI: 10.11622/smedj.2013126.

3. Thomas J, Fishel SB, Hall JA, et al. Increased polymorphonuclear granulocytes in seminal plasma in relation to sperm morphology. Hum Reprod Oxf Engl 1997;12(11):2418-2421. DOI: 10.1093/ humrep/12.11.2418.

4. Storey BT. Biochemistry of the induction and prevention of lipoperoxidative damage in human spermatozoa. Mol Hum Reprod 1997;3(3):203-213. DOI: 10.1093/molehr/3.3.203.

5. Aitken RJ, Gordon E, Harkiss D, et al. Relative impact of oxidative stress on the functional competence and genomic integrity of human spermatozoa. Biol Reprod 1998;59(5):1037-1046. DOI: 10.1095/ biolreprod59.5.1037.

6. Sukcharoen N, Keith J, Irvine DS, et al. Predicting the fertilizing potential of human sperm suspensions in vitro: importance of sperm morphology and leukocyte contamination. Fertil Steril 1995;63(6):1293-1300. DOI: 10.1016/S0015-0282(16)57614-6.

7. Lackner JE, Märk I, Sator K, et al. Effect of leukocytospermia on fertilization and pregnancy rates of artificial reproductive technologies. Fertil Steril 2008;90(3):869-871. DOI: 10.1016/j. fertnstert.2007.07.1310.

8. Daya $S$, Wikland M, Nilsson $L$, et al. Fertilization and embryo development of oocytes obtained transvaginally under ultrasound guidance. J In Vitro Fert Embryo Transf 1987;4(6):338-342. DOI: 10.1007/BF01555382.

9. Moubasher A, Sayed H, Mosaad E, et al. Impact of leukocytospermia on sperm dynamic motility parameters, DNA and chromosomal integrity. Cent Eur J Urol 2018;71(4):470-475.

10. Ricci G, Granzotto M, Luppi S, et al. Effect of seminal leukocytes on in vitro fertilization and intracytoplasmic sperm injection outcomes. Fertil Steril 2015;104(1):87-93. DOI: 10.1016/j.fertnstert.2015.04.007.

11. Cavagna M, Oliveira JBA, Petersen CG, et al. The influence of leukocytospermia on the outcomes of assisted reproductive technology. Reprod Biol Endocrinol RBE 2012;10(1):44. DOI: 10.1186/1477-7827-10-44.

12. Villegas J, Schulz M, Vallejos V, et al. Indirect immunofluorescence using monoclonal antibodies for the detection of leukocytospermia: comparison with peroxidase staining. Andrologia 2002;34(2):69-73. DOI: 10.1046/j.0303-4569.2001.00476.x.

13. Ricci G, Presani G, Guaschino S, et al. Leukocyte detection in human semen using flow cytometry. Hum Reprod OxfEngl 2000;15(6):13291337. DOI: 10.1093/humrep/15.6.1329. 
14. Brunner RJ, Demeter JH, Sindhwani P. Review of guidelines for the evaluation and treatment of leukocytospermia in male infertility. World J Mens Health 2019;37(2):128-137. DOI: 10.5534/wjmh.180078.

15. Punab M, Lõivukene K, Kermes K, et al. The limit of leucocytospermia from the microbiological viewpoint. Andrologia 2003;35(5):271-278. DOI: 10.1111/j.1439-0272.2003.tb00856.x.

16. Weidner W, Pilatz A, Diemer T, et al. Male urogenital infections: impact of infection and inflammation on ejaculate parameters. World J Urol 2013;31(4):717-723. DOI: 10.1007/s00345-013-1082-7.

17. Castellini C, D'Andrea S, Martorella A, et al. Relationship between leukocytospermia, reproductive potential after assisted reproductive technology, and sperm parameters: a systematic review and metaanalysis of case-control studies. Andrology 2020;8(1):125-135. DOI: 10.1111/andr.12662.

18. Agarwal A, Saleh RA, Bedaiwy MA. Role of reactive oxygen species in the pathophysiology of human reproduction. Fertil Steril 2003;79(4):829-843. DOI: 10.1016/S0015-0282(02)04948-8.

19. Ziyyat A, Barraud-Lange V, Sifer C, et al. Paradoxical increase of sperm motility and seminal carnitine associated with moderate leukocytospermia in infertile patients. Fertil Steril 2008;90(6):22572263. DOI: 10.1016/j.fertnstert.2007.10.032.

20. Lackner JE, Agarwal A, Mahfouz R, et al. The association between leukocytes and sperm quality is concentration dependent. Reprod Biol Endocrinol RBE 2010;8(1):12. DOI: 10.1186/1477-7827-8-12.

21. Erenpreiss J, Hlevicka S, Zalkalns J, et al. Effect of leukocytospermia on sperm DNA integrity: a negative effect in abnormal semen samples. J Androl 2002;23(5):717-723.
22. Salumets A, Suikkari AM, Möls T, et al. Influence of oocytes and spermatozoa on early embryonic development. Fertil Steril 2002;78(5):1082-1087. DOI: 10.1016/S0015-0282(02)04215-2.

23. Agarwal A, Makker K, Sharma R. Clinical relevance of oxidative stress in male factor infertility: an update. Am J Reprod Immunol N Y N 1989 2008;59(1):2-11.

24. Anderson DJ. Should male infertility patients be tested for leukocytospermia? Fertil Steril 1995;63(2):246-248. DOI: 10.1016/ S0015-0282(16)57348-8.

25. Sanocka D, Kurpisz M. Reactive oxygen species and sperm cells. Reprod Biol Endocrinol RBE 2004;2(1):12. DOI: 10.1186/1477-7827-2-12.

26. Rossi T, Mazzilli F, Delfino M, et al. Improved human sperm recovery using superoxide dismutase and catalase supplementation in semen cryopreservation procedure. Cell Tissue Bank 2001;2(1):9-13. DOI: 10.1023/A:1011592621487.

27. Cocuzza M, Sikka SC, Athayde KS, et al. Clinical relevance of oxidative stress and sperm chromatin damage in male infertility: an evidence based analysis. Int Braz J Urol Off J Braz Soc Urol 2007;33(5):603-621. DOI: 10.1590/S1677-55382007000500002.

28. Berkovitz A, Eltes F, Yaari S, et al. The morphological normalcy of the sperm nucleus and pregnancy rate of intracytoplasmic injection with morphologically selected sperm. Hum Reprod Oxf Engl 2005;20(1):185-190. DOI: 10.1093/humrep/deh545.

29. Barraud-Lange V, Pont J-C, Pocate K, et al. Seminal leukocytes and clinical outcomes with donor sperm insemination. Ferti Steril 2011;96(6):1320-1324.e1. DOI: 10.1016/j.fertnstert.2011. 08.025 